\title{
MINIMAL REARRANGEMENTS OF SOBOLEV FUNCTIONS: A NEW PROOF
}

\section{SUR LES RÉARRANGEMENTS EXTRÉMAUX DES FONCTIONS DE SOBOLEV: UNE NOUVELLE DÉMONSTRATION}

\author{
Adele FERONE ${ }^{\text {a }}$, Roberta VOLPICELLI ${ }^{\text {b,* }}$ \\ ${ }^{a}$ Dipartimento di Matematica, Seconda Università di Napoli, Via Vivaldi 43, Caserta, 81100, Italy \\ b Dipartimento di Matematica e Applicazioni "R. Caccioppoli", Università di Napoli "Federico II", \\ Complesso Monte S. Angelo, Via Cintia, Napoli, 80126, Italy
}

Received 9 July 2001, revised 11 November 2001

ABSTRACT. - We give an alternative proof of a theorem by Brothers and Ziemer concerning extremal functions in the Pólya-Szegö rearrangements inequality for Dirichlet type integrals. (C) 2003 L'Association Publications de l'Institut Henri Poincaré. Published by Elsevier B.V. All rights reserved

Keywords: Polar factorization; Rearrangements; Pólya-Szegö type inequalities

RÉSUMÉ. - Nous donnons une autre démonstration d'un théorème de Brothers et Ziemer qui concerne les fonctions extremales dans l'inégalité de Pólya-Szegö pour les intégrales de Dirichlet.

(C) 2003 L'Association Publications de l'Institut Henri Poincaré. Published by Elsevier B.V. All rights reserved

\section{Introduction}

Let $\Omega$ be an open, bounded subset of $\mathbb{R}^{N}$ and let $W_{0}^{1, p}(\Omega), 1 \leqslant p<+\infty$, be the Sobolev space of those functions whose extention by or outside $\Omega$ has weak derivatives summable to the power $p$ in $\mathbb{R}^{N}$.

The classical Pólya-Szegö principle states that if $u$ is a nonnegative function from $W_{0}^{1, p}(\Omega)$, then its spherically symmetric rearrangement $u^{\sharp}$ belongs to $W_{0}^{1, p}\left(\Omega^{\sharp}\right)$ and

$$
\left\|D u^{\sharp}\right\|_{L^{p}\left(\Omega^{\sharp}\right)} \leqslant\|D u\|_{L^{p}(\Omega)} .
$$

\footnotetext{
* Corresponding author.

E-mail addresses: adele.ferone@unina2.it (A. Ferone), roberta.volpicelli@dma.unina.it (R. Volpicelli).
} 
Here, $\Omega^{\sharp}$ is the ball in $\mathbb{R}^{N}$ centered at the origin and such that $\left|\Omega^{\sharp}\right|_{N}=|\Omega|_{N}$, where $|\cdot|_{N}$ denotes the Lebesgue measure in $\mathbb{R}^{N}$ (in the case $N=1$, we will omit the dimension), and $u^{\sharp}: \Omega^{\sharp} \rightarrow[0,+\infty)$ is defined as follows. Denote by $\mu_{u}$ the distribution function of $u$ given by

$$
\mu_{u}(t)=|\{x \in \Omega:|u(x)|>t\}|_{N} \quad \text { for } t \geqslant 0,
$$

and let $u^{*}$ be the decreasing rearrangement of $u$ defined by

$$
\left.\left.u^{*}(s)=\inf \left\{t \geqslant 0: \mu_{u}(t) \leqslant s\right\} \quad \text { for } s \in\right] 0,|\Omega|_{N}\right],
$$

then $u^{\sharp}$ is defined as

$$
u^{\sharp}(x)=u^{*}\left(\mathcal{C}_{N}|x|^{N}\right) \quad \text { for } x \in \Omega^{\sharp},
$$

where $\mathcal{C}_{N}$ is the measure of the $N$-dimensional unit ball. Formulated in [20], many authors gave proofs and generalizations of this principle (see, for instance, $[2,4-6,8,10$, 14,16-18,21,23-26]). Here, we deal with the problem of characterizing those functions for which equality holds in (1.1). Partial results are contained in [15] and [27]. The problem was also discussed by Friedman and McLeod in [13] when $u$ is of class $C^{n}$. However, as observed in [7], the proof in [13] contains an error which can be only repaired under additional assumptions. A general answer was given later by Brothers and Ziemer in [7], where the following theorem is proved.

THEOREM 1.1. - Let $\Omega$ be an open, bounded subset of $\mathbb{R}^{N}, N \geqslant 1$, and let $u$ be a nonnegative function from $W_{0}^{1, p}(\Omega), 1<p<+\infty$, such that

$$
\left|\left\{\left|D u^{\sharp}\right|=0\right\} \cap\left(u^{\sharp}\right)^{-1}(0, \operatorname{ess} \sup u)\right|_{N}=0 .
$$

If

$$
\left\|D u^{\sharp}\right\|_{L^{p}\left(\Omega^{\sharp}\right)}=\|D u\|_{L^{p}(\Omega)},
$$

then $\Omega$ is equivalent to a ball and $u=u^{\sharp}$ a.e. in $\Omega$, up to a translation.

If $p=1$, then the result is false, since every function $u$ from $W_{0}^{1,1}(\Omega)$ whose level sets are (not necessarily concentric) balls satisfies (1.3). Hypothesis (1.2) is equivalent to the absolute continuity of $\mu_{u}$ in $(0, \operatorname{ess} \sup u)$ (see [7, p. 157, Lemma 2.3]). If $\mu_{u}$ is not even assumed to be continuous in $(0, \operatorname{ess} \sup u)$, a situation occurring if and only if $|\{u=t\}|_{N}>0$ for some $t \in(0$, ess $\sup u)$, then simple counterexamples to the conclusion of the theorem can be constructed. A subtle counterexample involving a function $u$ whose distribution function $\mu_{u}$ is continuous but not absolutely continuous is produced in [7].

The proof of Theorem 1 in [7] can be split into two steps. The first one consists in showing that, for a.e. $t \in(0$, ess $\sup u)$, the level sets $\{u>t\}$ are equivalent to balls and that on their boundaries $|D u|=\left|D u^{\sharp}\right|_{\left\{u^{\sharp}=t\right\}}$, a constant depending only on $t$. This step does not require (1.2) and its proof is based on the observation that all the inequalities over the level sets of $u$ which lead to (1.1) must hold as equations in the case where (1.3) is in force. Basic tools here are the isoperimetric theorem and the coarea formula. Proofs of this part can be found also elsewhere (see [13,27]). 
The really delicate task in the proof of Theorem 1 is the second step, where the balls $\{u>t\}$ are shown to be concentric. Even if based on a geometrically clear approach, the rigorous justification of the arguments of [7] for this part is accomplished after overcoming serious technical difficulties by means of results from geometric measure theory.

The aim of this note is to give an alternative proof of this second step, based on the explicit relation between $u$ and $u^{*}$, given by

$$
u(x)=u^{*}\left(\mu_{u}(u(x))\right) \quad \text { for } x \in \Omega,
$$

and on arguments from the classical theory of Sobolev spaces, that eventually allow us to apply the method of the steepest descent introduced by Aronsson and Talenti in [3].

\section{Proof of Theorem 1}

As pointed out in the introduction, the first step in the proof of Theorem 1 is the following characterization of those functions that verify (1.3) (see [7, p. 161, Lemma 3.1]).

LEMMA 2.1. - Let $1 \leqslant p<+\infty$ and let $u \in W_{0}^{1, p}(\Omega)$ be a nonnegative function satisfying

$$
\left\|D u^{\sharp}\right\|_{L^{p}\left(\Omega^{\sharp}\right)}=\|D u\|_{L^{p}(\Omega)} .
$$

Then

$$
\mathcal{H}_{N-1}(\{x: u(x)=t\})=\mathcal{H}_{N-1}\left(\left\{x: u^{\sharp}(x)=t\right\}\right) \quad \text { for a.e. } t \in(0, \text { ess sup } u),
$$

and, if $p>1$, then

$$
|D u|(x)=\left|D u^{\sharp}\right| \quad \text { for } \mathcal{H}_{N-1} \text {-a.e. } x \in\{y: u(y)=t\} .
$$

Here, $\left|D u^{\sharp}\right|$ denotes the constant value of $\left|D u^{\sharp}\right|$ on $\left\{x: u^{\sharp}(x)=t\right\}$ and $\mathcal{H}_{N-1}$ denotes $(N-1)$-dimensional Hausdorff measure.

Eq. (2.1) and the Isoperimetric Theorem of De Giorgi [9] imply that for a.e. $t \in$ $(0$, ess $\sup u)$, and hence for every $t \in(0, \operatorname{ess} \sup u)$, the sets $\{x: u(x)>t\}$ are equivalent to balls. In particular $\Omega$ is a ball, that without loss of generality, we will suppose to be centered at the origin; namely $\Omega=\Omega^{\sharp}$, up to set of measure zero.

In the following we will suppose, without loss of generality, that $|\{u=\operatorname{ess} \sup u\}|_{N}=$ $|\{u=0\}|_{N}=0$.

Our aim is to accomplish the proof of Theorem 1 on applying the method of the steepest descent introduced by Aronsson and Talenti in [3]. This method cannot be applied directly to $u$, since $u$ is not Lipschitz continuous. Nevertheless, since $u$ satisfies (1.2), then its distribution function $\mu_{u}$ is a one-to-one function on $(0, \operatorname{ess} \sup u)$ which is absolutely continuous. This fact allows us to regard the level sets of $u$ as the level sets of a Lipschitz continuous function to which we can apply the method of the 
steepest descent. Indeed, define the function

$$
\sigma(x)=\mu_{u}(u(x)) \text { for a.e. } x \in \Omega^{\sharp} .
$$

Since $\mu_{u}$ is a one-to-one function on $(0, \operatorname{ess} \sup u)$ and $u^{*}$ is its inverse, then $u^{*}\left(\mu_{u}(t)\right)=t$ for every $t \in(0, \operatorname{ess} \sup u)$. Hence,

$$
u(x)=u^{*}(\sigma(x)) \text { for a.e. } x \in \Omega^{\sharp} .
$$

The functions $u$ and $\sigma$ have the same level sets, in the sense that, for every $t \in$ $(0$, ess $\sup u)$ there exists $\tau \in\left(0,|\Omega|_{N}\right)$, such that

$$
\{x: u(x)>t\}=\{x: \sigma(x)<\tau\} \quad \text { up to set of measure zero. }
$$

Furthermore, $\sigma$ is a measure preserving map, in the sense that, for every measurable subset $A$ of $\left[0,|\Omega|_{N}\right]$, we have

$$
\left|\sigma^{-1}(A)\right|_{N}=|A| \text {. }
$$

Indeed, it is easily verified that $\sigma$ has the same distribution function as the identity map on $\left[0,|\Omega|_{N}\right]$. Namely, $\left|\sigma^{-1}\left(t,|\Omega|_{N}\right)\right|_{N}=|\Omega|_{N}-t$. Therefore $\left|\sigma^{-1}(a, b)\right|_{N}=b-a$, for every $a, b \in\left[0,|\Omega|_{N}\right], a<b$, which by a limiting argument clearly implies (2.5) (see [22]). As a consequence of (2.5), we get that for every measurable subset $A$ of $\mathbb{R}$ such that $|A|=0,\left|u^{-1}(A)\right|_{N}=0$. Actually, let $A$ be a measurable subset of $\mathbb{R}$ such that $|A|=0$, then by (2.3), $x \in u^{-1}(A)$ if and only if $\sigma(x) \in \mu_{u}(A)$.

Thus, by (2.5) and by the absolutely continuity of $\mu_{u}$, we have

$$
\left|u^{-1}(A)\right|_{N}=\left|\sigma^{-1}\left(\mu_{u}(A)\right)\right|_{N}=\left|\mu_{u}(A)\right|=0 .
$$

In the last equation, we have used the fact that the image of a set of measure zero by an absolutely continuous function has measure zero. Property (2.6) is needed to show that $\sigma \in W^{1, \infty}\left(\Omega^{\sharp}\right)$. Indeed $\sigma \in L^{\infty}\left(\Omega^{\sharp}\right)$, since $\mu_{u} \in L^{\infty}(0$, ess sup $u)$. Therefore, in order to prove that $\sigma$ is Lipschitz continuous, it remains to prove that the restriction of $\sigma$ to almost every straight line parallel to the coordinate axes is absolutely continuous and that $|D \sigma| \in L^{\infty}\left(\Omega^{\sharp}\right)$ (see [19]).

First we prove that the restriction of $\sigma$ to almost every straight line parallel to a coordinate axes, has bounded variation. Let $\bar{y} \in \mathbb{R}^{N-1}$ be such that, if $\ell$ is the straight line defined as $\ell=\{(t, \bar{y}): t \in \mathbb{R}\}$, then $\ell \cap \Omega^{\sharp} \neq \emptyset$ and let $\sigma_{\mid \ell}$ be the restriction of $\sigma$ to $\ell$. Since the level sets of $u$ are balls, then there exists $\bar{t} \in \mathbb{R}$ such that, $u(t, \bar{y})$ is strictly increasing for $t \leqslant \bar{t}$ and strictly decreasing for $t>\bar{t}$. This implies that, on denoting by $V\left(\sigma_{\mid \ell}\right), V_{-\infty}^{\bar{t}}\left(\sigma_{\mid \ell}\right)$ and $V_{\bar{t}}^{+\infty}\left(\sigma_{\mid \ell}\right)$ the variations of $\sigma_{\mid \ell}$ on $\mathbb{R},(-\infty, \bar{t})$ and $(\bar{t},+\infty)$, respectively, then

$$
V\left(\sigma_{\mid \ell}\right)=V_{-\infty}^{\bar{t}}\left(\sigma_{\mid \ell}\right)+V_{\bar{t}}^{+\infty}\left(\sigma_{\mid \ell}\right)=2 \mid\left\{u(\cdot, \bar{y}) \leqslant\left. u(\bar{t}, \bar{y})\right|_{N} \leqslant 2\left|\Omega^{\sharp}\right|_{N} .\right.
$$

Analogously, one can deduce that $\sigma$ has bounded variation on almost all straight lines which are parallel to the other coordinate axes. 
Since $u \in W_{0}^{1, p}\left(\Omega^{\sharp}\right)$, then the restriction of $u$ to almost every straight line parallel to the coordinate axes is absolutely continuous (see [19]). Then, also the restriction of $\sigma$ to almost every straight line parallel to the coordinate axes is absolutely continuous, since it is the composition of two absolutely continuous functions and it has bounded variation.

Therefore, if $D \sigma$ is the usual gradient of $\sigma$, applying the chain rule to (2.3), we get

$$
D \sigma(x)=\mu_{u}^{\prime}(u(x)) D u(x) \text { for a.e. } x \in \Omega^{\sharp} \backslash u^{-1}(I),
$$

where $I=\left\{t\right.$ : does not exists $\left.\mu_{u}^{\prime}(t)\right\}$. Since $|I|=0$, we deduce, by (2.6) that $\left|u^{-1}(I)\right|_{N}$ $=0$. Hence, (2.7) holds for a.e. $x \in \Omega^{\sharp}$.

On the other hand, by the coarea formula (see $[11,12]$ ), we have that

$$
-\mu_{u}^{\prime}(t)=\frac{\mathcal{H}_{N-1}\left(\left\{x: u^{\sharp}(x)=t\right\}\right)}{\left|D u^{\sharp}\right|_{\left\{u^{\sharp}=t\right\}}} \quad \text { for a.e. } t \in(0, \text { ess } \sup u)
$$

where $\left|D u^{\sharp}\right|_{\left\{u^{\sharp}=t\right\}}$ is the constant value of $\left|D u^{\sharp}\right|$ on the set $\left\{x: u^{\sharp}(x)=t\right\}$.

Since the level set $\left\{x: u^{\sharp}(x)=t\right\}$ is the boundary of the ball $\left\{x: u^{\sharp}(x)>t\right\}$ whose measure is $\mu_{u}(t)$, by (2.2) we have

$$
-\mu_{u}^{\prime}(t)=\frac{N \mathcal{C}_{N}^{1 / N} \mu_{u}(t)^{1-1 / N}}{|D u|_{\{u=t\}}} \quad \text { for a.e. } t \in(0, \operatorname{ess} \sup u)
$$

where $|D u|_{\{u=t\}}$ is the constant value of $|D u|$ on the set $\{x: u(x)=t\}$.

Combining (2.7) and (2.8), yields

$$
D \sigma(x)=-N \mathcal{C}_{N}^{1 / N} \mu_{u}(u(x))^{1-1 / N} \frac{D u(x)}{|D u(x)|} \quad \text { for a.e. } x \in \Omega^{\sharp}
$$

hence, by the definition of $\sigma$,

$$
|D \sigma(x)|=N \mathcal{C}_{N}^{1 / N} \sigma(x)^{1-1 / N} \quad \text { for a.e. } x \in \Omega^{\sharp} .
$$

By (2.9) and by the fact that $\sigma \in L^{\infty}\left(\Omega^{\sharp}\right)$, we deduce that $|D \sigma| \in L^{\infty}\left(\Omega^{\sharp}\right)$.

Let us, now, consider the function $F$ defined as

$$
F(x)=\mathcal{C}_{N}^{-1 / N} \sigma(x)^{1 / N} \text { for } x \in \Omega^{\sharp} .
$$

The function defined above is that one we are looking for, in order to apply the method of the steepest descent, introduced by Aronsson and Talenti in [3] (see also [1]). By (2.4) $F$ and $u$ have the same level sets in the sense specified above. Furthermore, by (2.9), $|D F|=1$ a.e. in $\Omega^{\sharp}$, and then $F \in W^{1, \infty}\left(\Omega^{\sharp}\right)$. Let $s \rightarrow x(s)$ be any solution to the dynamical system $\frac{\mathrm{d} x}{\mathrm{~d} s}=X(x)$, where $X(x)$ is the outer normal to $\partial\left\{y \in \Omega^{\sharp}: F(y)<\right.$ $F(x)\}$ at the point $x$, or equivalently, to $\partial\left\{y \in \Omega^{\sharp}: u(y)>u(x)\right\}$ (notice that, under our assumption, $x \in \partial\left\{y \in \Omega^{\sharp}: u(y)>u(x)\right\}$ for a.e. $x \in \Omega^{\sharp}$ ). The fact that $\partial\{y \in$ $\left.\Omega^{\sharp}: F(y)<F(x)\right\}$ is a sphere ensures that $X$ is locally Lipschitz continuous, this implies the local existence and uniqueness of the line $x=x(s)$ defined as above. Moreover 
$X(x)=D F(x)$ if $F$ is differentiable at $x$; for this reason, we say that the lines $x=x(s)$, are the lines of steepest descent of $F$. We want to prove that such lines are straight. Since $\left|\frac{\mathrm{d} x}{\mathrm{~d} s}\right|=|X(x(s))|=1$ then $s$ is the arclenght. Therefore,

$$
\frac{\mathrm{d}}{\mathrm{d} s} F(x(s))=\left\langle D F(x(s)), \frac{\mathrm{d} x}{\mathrm{~d} s}(s)\right\rangle=\langle X(x(s)), X(x(s))\rangle=1 .
$$

Thus,

$$
\left|s_{2}-s_{1}\right|=\left|\int_{s_{1}}^{s_{2}} \frac{\mathrm{d}}{\mathrm{d} s} F(x(s)) \mathrm{d} s\right|=\left|F\left(x\left(s_{2}\right)\right)-F\left(x\left(s_{1}\right)\right)\right| .
$$

On the other hand, since $F$ is Lipschitz continuous and $|D F| \equiv 1$, then

$$
\left|F\left(x\left(s_{2}\right)\right)-F\left(x\left(s_{1}\right)\right)\right| \leqslant\left|x\left(s_{2}\right)-x\left(s_{1}\right)\right| .
$$

From (2.10) and (2.11) we get

$$
\left|s_{2}-s_{1}\right| \leqslant\left|x\left(s_{2}\right)-x\left(s_{1}\right)\right|,
$$

that is the lenght of the arc is less or equal then the lenght of the chord through $x\left(s_{1}\right)$ and $x\left(s_{2}\right)$. Hence, the line in question must be straight. This implies that the level sets of $F$, and hence the level sets of $u$, are concentric balls, therefore $u=u^{\sharp}$ up to set of measure zero.

\section{Acknowledgement}

We would like to thank Professors A. Cianchi and N. Fusco for some helpful discussions.

\section{REFERENCES}

[1] A. Alvino, P.L. Lions, G. Trombetti, A remark on comparison result via Schwartz symmetrization, Proc. Roy. Soc. Edinburgh. 102-A (1-2) (1986) 37-48.

[2] A. Alvino, V. Ferone, P.L. Lions, G. Trombetti, Convex symmetrization and applications, Ann. Inst. H. Poincaré, Anal. Non Linéaire 14 (2) (1997) 275-293.

[3] G. Aronsson, G. Talenti, Estimating the integral of a function in terms of a distribution function of its gradient, Boll. Un. Mat. Ital. (5) 18-B (3) (1981) 885-894.

[4] T. Aubin, Problèmes isopérimétriques et espaces de Sobolev, C. R. Acad. Sci. Paris 280 (1975) 279-281.

[5] M.F. Betta, F. Brock, A. Mercaldo, M.R. Posteraro, A weighted isoperimetric inequality and applications to symmetrization, J. Inequal. Appl. 4 (3) (1999) 215-240.

[6] F. Brock, Weighted Dirichlet-type inequalities for Steiner symmetrization, Calc. Var. Partial Differential Equations 8 (1999) 15-25.

[7] J.E. Brothers, W.P. Ziemer, Minimal rearrangements of Sobolev functions, J. Reine Angew. Math. 384 (1988) 153-179.

[8] A. Cianchi, L. Pick, Sobolev embeddings into BMO, VMO and $L^{\infty}$, Ark. Mat. 36 (2) (1998) $317-340$. 
[9] E. De Giorgi, Su una teoria generale della misura $(r-1)$-dimensionale in uno spazio ad $r$ dimensioni, Ann. Mat. Pura Appl. 36 (4) (1954) 191-213.

[10] G.F.D. Duff, A general integral inequality for the derivative of an equimeasurable rearrangement, Canad. J. Math. 28 (4) (1976) 793-804.

[11] H. Federer, Geometric Measure Theory, Springer, Berlin, 1969.

[12] W. Fleming, R. Rishel, An integral formula for total gradient variation, Arch. Math. 11 (1960) 218-222.

[13] A. Friedman, R. McLeod, Strict inequalities for integrals of decreasingly rearranged functions, Proc. Roy. Soc. Edinburgh 102-A (3-4) (1986) 277-289.

[14] K. Hilden, Symmetrization of functions in Sobolev spaces and the isoperimetric inequality, Manuscripta Math. 18 (3) (1976) 215-235.

[15] B. Kawohl, Rearrangements and Convexity of Level Sets in P.D.E., in: Lecture Notes in Math., Vol. 1150, Springer, Berlin, 1985.

[16] V.S. Klimov, Imbedding theorems and geometric inequalities, Izv. Akad. Nauk USSR Ser. Mat. 40 (3) (1976) 645-671.

[17] V.M. Maz'ja, Sobolev Spaces, Springer, Berlin, 1985.

[18] J. Mossino, Inégalités Isopérimétriques et Applications en Physic, in: Collection Travaux en Cours, Hermann Paris, 1984.

[19] C.B. Morrey, Multiple Integrals in the Calculus of Variations, Springer, Berlin, 1966.

[20] G. Pólya, G. Szegö, Isoperimetric Inequalities in Mathematical Physics, in: Ann. of Math. Studies, Vol. 27, Princeton University Press, Princeton, 1951.

[21] J.M. Rakotoson, R. Temam, A co-area formula with applications to monotone rearrangement and to regularity, Arch. Rational Mech. Anal. 109 (3) (1990) 213-238.

[22] J.V. Ryff, Measure preserving transformations and rearrangements, J. Math. Anal. Appl. 31 (3) (1970) 449-458.

[23] E. Sperner, Zur Symmetrisierung von Funktionen auf Sphären, Math. Z. 134 (1973) 317327.

[24] E. Sperner, Symmetrisierung für Funktionen mehrerer reeler Variablen, Manuscripta Math. 11 (1974) 159-170.

[25] G. Talenti, Best constant in Sobolev inequality, Ann. Mat. Pura Appl. Cl. Sci. (4) 110 (1976) 353-372.

[26] G. Talenti, A weighted version of a rearrangement inequality, Ann. Univ. Ferrara 43 (1997) $121-133$.

[27] A. Uribe, Minima of Dirichlet norm and Topeliz operators, Preprint, 1985. 